Copyright (C1996, American Institute of Aeronautics and Astronautics, Inc.

AIAA Meeting Papers on Disc, June 1996

A9636348, F49620-94-1-0019, F49620-95-1-0405, AIAA Paper 96-1856

\title{
Nonequilibrium real-gas effects on disturbance/bow shock interaction in hypersonic flow past a cylinder
}

\author{
Xiaolin Zhong \\ California Univ., Los Angeles \\ Theodore K. Lee \\ California Univ., Los Angeles
}

\begin{abstract}
AIAA, Thermophysics Conference, 31st, New Orleans, LA, June 17-20, 1996
The interaction of free-stream disturbances and the bow shock wave in hypersonic flow past a blunt leading edge is an important part of hypersonic boundary layer receptivity to free-stream disturbances. It has been shown that, for a perfect gas, the back and forth interaction and reflection of acoustic waves behind the shock greatly amplify the magnitudes of the disturbance waves. The interaction also generates a wide range of wavelengths for entropy and vorticity waves behind the shock due to the nonuniformity of mean flow over the body. For hypersonic flow over a blunt body, real gas effects become important because of high gas temperature behind the shock. In this paper, nonequilibrium real gas effects on the free-stream disturbance/bow shock interaction for hypersonic flow past a cylinder are investigated by numerical simulations and by linear analysis. The freestream disturbances are fixed-frequency planar acoustic wave. It was found that the real gas effects reduce the intensity of the space-time focusing of entropy waves on the wall and introduce new small length scales related to vibrational relaxation to the interaction wave fields. (Author)
\end{abstract}




\title{
NONEQUILIBRIUM REAL-GAS EFFECTS ON DISTURBANCE/BOW SHOCK INTERACTION IN HYPERSONIC FLOW PAST A CYLINDER
}

\author{
Xiaolin Zhong *and Theodore K. Lee ${ }^{\dagger}$ \\ University of California, Los Angeles, California 90095
}

\begin{abstract}
The interaction of free-stream disturbances and the bow shock wave in hypersonic flow past a blunt leading edge is an important part of hypersonic boundary layer receptivity to free-stream disturbances. It has been shown that, for a perfect gas, the back and forth interaction and reflection of acoustic waves behind the shock greatly amplify the magnitudes of the disturbance waves. The interaction also generates a wide range of wavelengths for entropy and vorticity waves behind the shock due to the nonuniformity of mean flow over the body. For hypersonic flow over a blunt body, real gas effects become important because of high gas temperature behind the shock. In this paper, nonequilibrium real gas effects on the free-stream disturbance/bow shock interaction for hypersonic flow past a cylinder are investigated by numerical simulations and by linear analysis. The freestream disturbances are fixed-frequency planar acoustic wave. It was found that the real gas effects reduce the intensity of the space-time focusing of entropy waves on the wall and introduce new small length scales related to vibrational relaxation to the interaction wave fields.
\end{abstract}

\section{Introduction}

The bow-shock/disturbance interaction problem originates from the studies of the receptivity of hypersonic boundary layers to free-stream disturbances. The receptivity, which refers to the processes by which the environmental disturbances initially enter the boundary layers and generate linear instability waves ${ }^{[1]}$, is an important aspect of transition from laminar to turbulent states of boundary layers. For hypersonic flow past a blunt leading edge, the bow shock wave has strong effects on the stability and transition of the boundary layer behind the shock. The curved bow shock cre-

\footnotetext{
*Assistant Professor, Mechanical and Aerospace Engineering Department, Member AIAA.

$\dagger_{\text {Graduate Student, Mechanical and Aerospace Engineering }}$ Department.

Copyright (C)1996 by American Institute of Aeronautics and Astronautics, Inc. All rights reserved.
}

ates entropy and vorticity layers, which are eventually swallowed by the boundary layer.

Figure 1 shows a schematic of the interaction of the bow shock over a blunt body and a freestream disturbance wave. Kovasznay ${ }^{[2]}$ showed that weak disturbance waves in compressible flow can be decomposed into three independent modes: acoustic mode, entropy mode, and vorticity mode. The wave of the acoustic mode is propagated with the speed of sound relative to the moving fluid, while the waves of the entropy mode and vorticity mode convect with the moving fluid velocity. Irrespective of the nature of the freestream disturbance wave, its interaction with the bow shock always generates all three types of disturbance waves. If the amplitude of the freestream wave is small, the three transmitted waves are propagated downstream independently, connected only by the jump conditions across the shock and the boundary conditions at the wall. The effects of the body are represented by upstream-propagated acoustic waves, which perturb the bow shock from behind and are rereflected from the shock as shown in Fig. 1. These back-and-forth acoustic wave reflections and interactions between the bow shock and the body cause considerable changes in all three disturbance waves behind the shock and raise the question of whether the bow shock wave is stable in such interactions.

The interaction of disturbance waves and shock waves without body effects has been studied extensively in acoustic research and turbulence research. For example, the acoustic wave generations by the linearized interaction of fixed-wavelength planar disturbance waves and shock waves were studied by Ribner [3], by Chang [4, 5], and by McKenzie and Westphal [6]. These linear analyses have shown that the interaction always generates all three types of disturbance waves behind the shock. The interactions of freestream turbulence with a planar shock wave have been studied by linearized analysis and by direct numerical simulations. Ribner [7], Anyiwo and Bushnell ${ }^{[8]}$, and more recently Lele ${ }^{[9]}$ did the linearized analyses. Direct numerical simulations were conducted by Zang, Hussaini, and Bushnel[ ${ }^{[10]}$, by Hussaini, Collier, and Bushnell ${ }^{[11]}$, by Rotman ${ }^{[12]}$, and by Lee, Lele, and Moin ${ }^{[13,14]}$. 
For the interaction of freestream disturbances and the bow shock in hypersonic flow past a cylinder, Chiu and Zhong ${ }^{[15]}$ and Zhong et al. ${ }^{[16]}$ did linear analysis and two-dimensional numerical simulations of the Euler equations with a perfect gas assumption. The freestream disturbances were plane acoustic waves and entropy waves of fixed-wavelengths. The effects of the blunt body and the nonuniform mean flow behind the shock to the disturbance wave fields were investigated. It was found that the pressure, density, and vorticity perturbations generated behind the shock are considerably amplified by wave interaction between the bow shock and the body. It was also found that the nonuniformity of the steady background mean flow between the bow shock and the body causes a space-time focusing ${ }^{[17]}$ of the entropy and vorticity waves on the body surface. As a result, the Euler solutions of the entropy and vorticity waves are singular on the body surface. Though incoming disturbance wave has a single wave length in the freestream, there are a wide range of length scale for entropy and vorticity waves, and their local wavelengths behind the shock gradually reduce to zero at the stagnation point. This reduction of length scales of disturbance waves on the wall can be important to the studies of the stability and transitions of hypersonic boundary layers because it is these waves with reduced wavelengths which will interact with the boundary layer downstream.

In the studies of [15] and [16], the hypersonic flow was assumed to be a perfect gas. For practical hypersonic flow past a blunt body, however, the real gas effects are often important because of high gas temperature behind the bow shock. The real gas effects that are first excited as temperature increases are the vibrational excitation and chemical dissociation and recombination for diatomic molecules. These real gas effects can have significant impact on the stability of the boundary layers behind the shock. In addition, the real gas effects ${ }^{[18]}$ further increase the intensity of entropy and vorticity layers created by the curved bow shock. As the entropy and vorticity layers convect downstream, they are "swallowed" by the boundary layers. The real gas effects also introduce to the flow fields new time and length scales related to the relaxation processes of the gas internal modes. A flow field can be classified as equilibrium, nonequilibrium, and frozen flow according to the magnitudes the relaxation length scales with respect to the macroscopic characteristic length. So far, the real-gas effects on the stability of hypersonic boundary layer with parallel assumption were studied by Malik ${ }^{[19]}$, Stuckert and Reed ${ }^{[20]}$ and Hudson et al. ${ }^{[21]}$. The real-gas effects on the stability and transition of the hypersonic boundary layer over a blunt leading edge has not been studied.
The purpose of this paper is to investigate, by both numerical simulations and linear analysis, the nonequilibrium real gas effects on the interaction between the bow shock and freestream disturbances in two-dimensional hypersonic flow past a cylinder. The freestream disturbance waves are assumed to be fixedwavelength planar acoustic waves. The governing equations are the Euler equations with finite rate chemical reactions and vibration nonequilibrium modes. Highorder finite-difference shock capturing schemes are used in the numerical simulations. The stiffness of the reacting flow equations are removed by using a third-order semi-implicit Runge-Kutta methods ${ }^{[22]}$. The effects of internal-mode relaxation length scales to the wave field generated by the shock/disturbance wave interaction are studied by using the hypersonic flow past three cylinders with the same freestream flow conditions but different cylinder radius. The flow is assumed to be inviscid in the present work. The viscous effects in the boundary layer will be the subject of future investigations. Meanwhile, the real gas effects are also examined by extending the approximate linear analyses of McKenzie and Westphal ${ }^{[6]}$ and the approximate approach of Morkovin ${ }^{[1]}$ to equilibrium real-gas flow behind the shock to study the transfer relations of disturbance waves across a local section of the bow shock with and without body effects.

\section{Governing Equations and Real-Gas Models}

The real gas models used in this paper for nonequilibrium hypersonic flow without ionization and radiation are the 5 -species nonequilibrium air model by Park ${ }^{[23,24]}$. The models are appropriate for flow temperature below $9000^{\circ} \mathrm{K}$. The governing equations are the following Euler equations with finite rate source terms:

$$
\frac{\partial \mathbf{U}}{\partial t}+\frac{\partial \mathbf{F}_{j}}{\partial x_{j}}=\mathbf{W}
$$

where

$$
\mathbf{U}=\left\{\begin{array}{c}
\rho_{1} \\
\vdots \\
\rho_{m} \\
\rho u_{1} \\
\rho u_{2} \\
e \\
e_{v}
\end{array}\right\}, \quad \mathbf{W}=\left\{\begin{array}{c}
\dot{w}_{1} \\
\vdots \\
\dot{w}_{m} \\
0 \\
0 \\
0 \\
\dot{w}_{v}
\end{array}\right\}
$$




$$
\mathbf{F}_{j}=\left\{\begin{array}{c}
\rho_{1} u_{j} \\
\vdots \\
\rho_{m} u_{j} \\
\rho u_{1} u_{j}+p \delta_{1 j} \\
\rho u_{2} u_{j}+p \delta_{2 j} \\
(e+p) u_{j} \\
e_{v} u_{j}
\end{array}\right\}
$$

where $\mathbf{F}_{j}$ is the inviscid flux vectors in $j$-th coordinate direction, and $\mathbf{W}$ is the vector for source terms of the nonequilibrium vibrational and chemical processes.

In the model, translational and rotational modes are assumed to have a single translational-rotational temperature $T$, and the vibrational modes of all diatomic species are modeled by a single vibrational temperature $T_{v}$. The pressure is

$$
p=\sum_{i}^{m} \rho_{i} R_{i} T
$$

where $R_{i}$ is the species specific gas constant. The total energy is

$$
e=\sum_{i=1}^{m} \rho_{i}\left(c_{v i} T+h_{i}^{0}\right)+e_{v}+\frac{\rho}{2} u_{k} u_{k}
$$

where $h_{i}^{0}$ is the heats of formation, and $c_{v i}$ is the specific heat at constant volume, which is equal to $3 R_{i} / 2$ and $5 R_{i} / 2$ for monatomic and diatomic species respectively. The total vibrational energy is

$$
e_{v}=\sum_{i}^{m_{d}} \rho_{i} R_{i} \frac{\theta_{v i}}{e^{\theta_{v i} / T_{v}}-1}
$$

where the summation is carried out only for diatomic species that are listed as the first $m_{d}$ of $m$ species, and $\theta_{v i}$ is the characteristic vibrational temperature for a species.

In the governing equations, $\dot{w}_{i}$ is the chemical source terms and $\dot{w}_{v}$ is the vibrational source term. The reaction equations for the current five-species model of Park $^{[23]}$ are

$$
\begin{aligned}
N_{2}+M & \rightleftharpoons 2 N+M \\
O_{2}+M & \rightleftharpoons 2 O+M \\
N O+M & \rightleftharpoons N+O+M \\
N_{2}+O & \rightleftharpoons N O+N \\
N O+O & \rightleftharpoons O_{2}+N
\end{aligned}
$$

where $\mathrm{M}$ denotes any of the species. The details of the formulas for $\dot{w}_{i}$ and the reaction rates can be found in [23]. The source term for the vibrational nonequilibrium for the translation-vibration coupling is modeled by the Landau-Teller model as

$$
Q_{T-V}=\rho_{s} \frac{e_{v s}(T)-e_{v s}\left(T_{v}\right)}{\tau_{s}}
$$

where

$$
e_{v s}(T)=\frac{R_{s} \theta_{s}}{e^{\theta_{v} T}-1}
$$

and $\tau_{s}$ is the relaxation time given by Millikan and White ${ }^{[25]}$.

\section{Numerical Methods}

The Euler equations are solved by finite difference upwind schemes using the approach of Shu and Osher for the finite-difference implementation of the ENO schemes [26]. Both high-order ENO and TVD schemes ([27], [28]) for solving the Euler equations are used for the spatial discretization of the Euler equations. The semi-discrete equations are then advanced in time using the third-order accurate semi-implicit TVD Runge-Kutta schemes ${ }^{[2]}$ to remove the stiffness of the nonequilibrium equations. Details of the numerical methods and test results on numerical accuracy of the numerical methods for the shock/disturbance interaction problem can be found in $[15,16]$. The boundary conditions in the free stream are specified to be the given free-stream values. Those in the supersonic exit are calculated by an extrapolation method. For the inviscid flow computations, characteristic equations are used to compute the boundary conditions at the wall.

\section{Bow Shock/Disturbance Interaction Test Cases}

The interaction of incident acoustic waves and the bow shock over the cylinder are studied by timeaccurate numerical simulations of the Euler equations with vibrational and chemical nonequilibrium. Before impinging on the shock, the incident plane acoustic waves are propagated horizontally with a given frequency. The effects of thermal and chemical relaxation are studied by numerical simulations. Meanwhile, grid refinement studies are used to evaluate the accuracy of the numerical results.

The mean flow conditions of the test cases are chosen to be the same as the experimental measurement for 
steady hypersonic flow past a cylinder by Hornung ${ }^{[29]}$. The freestream mean-flow conditions are

$$
\begin{array}{ll}
C_{N_{2}}=0.927, & C_{N}=0.073, \\
u_{\infty}=5590 \mathrm{~m} / \mathrm{s}, & T_{\infty}=1833 \mathrm{~K}, \\
p_{\infty}=2910 \mathrm{~Pa}, &
\end{array}
$$

where $C_{N_{2}}$ and $C_{N}$ are the mass fraction of $N_{2}$ and $N$. For the same freestream conditions, three test cases with different cylinder radiuses are computed to investigate the effects of nonequilibrium time and length scales on the wave fields generated by the unsteady shock/disturbance interaction and wall reflection, i.e.,

Case A: $r=1.27 \times 10^{-3} \mathrm{~m}$

(Nonequilibrium but near frozen flow limit)

Case B: $r=5.08 \times 10^{-3} \mathrm{~m}$

(Nonequilibrium flow)

Case C: $r=2.54 \times 10^{-2} \mathrm{~m}$

(Nonequilibrium but near equilibrium limit)

where $r$ is the cylinder radius. Because $r$ is small in Case $\mathrm{A}$, the internal relaxation length at the point immediately behind the shock is larger than the macroscopic length. Therefore, the flow is near frozen flow in most part of the flow field behind the shock for Case A. As $r$ becomes larger, the flow fields are more equilibrium in Case B, and is near equilibrium for Case C. Among the three test cases, the differences in the results are mainly due to the differences in the degrees of nonequilibrium in the flow fields.

For hypersonic flow over the cylinder, the steady flow is not uniform in the degrees of nonequilibrium behind the bow shock. Though the flow is near frozen for flow behind the bow shock for Case $A$, the flow velocity reduces to zero at the stagnation point. As a result, the flow reaches equilibrium as it slowly approaches the stagnation point. In addition, the flow is not uniform behind the curved bow shock because the strength of the bow shock decreases as the shock location moves away from the stagnation line. The flow variables and the degrees of nonequilibrium change accordingly. The effects of the nonuniformity of the nonequilibrium flow behind the shock is an important aspect of characteristics of the wave fields between the bow shock and the body.

In this paper, the interaction of the bow shock and incident planar acoustic waves for hypersonic flow over a cylinder are studied by numerical simulations. The numerical simulations of the interaction are carried out in three steps. First, the steady mean flow field is computed. Second, periodic perturbations are imposed on the free stream of the mean flow variables and the timeaccurate solutions of bow shock/disturbance interaction are advanced in time for several periods until the wave field reaches periodic states. Third, an additional period in time is computed for the root-mean-square (RMS) values of the perturbation variables in the flow field.

\section{Mean Flow Solutions}

The results in this paper are obtained using uniform $120 \times 240$ body fitted grids. Only the upper half of the flow field is computed because of symmetry. Figure 7 shows the density contours of the mean flow of the three test cases of different degree of nonequilibrium. In the figures, the geometrical coordinates of all the cases are normalized by their respective cylinder radiuses. The results show that as the flows change from near frozen flow of Case A to near equilibrium flow of Case $\mathrm{C}$, the standoff distance decreases, and the density increases.

Figures 3 to 5 show the distributions of density, pressure, and $N_{2}$ mass fraction along the stagnation line for the three test cases. In these figures, the $x$ coordinate is normalized by the standoff distance in order to compare the results of of the test cases. The results show that as the radius increases from case $\mathrm{A}$ to Case $\mathrm{C}$, the flows become more and more equilibrium which can be seen in the figures through the increase of the density and more $N_{2}$ dissociation behind the shocks. But the pressure distribution is much less affected by the degree of nonequilibrium in the flow field. The figures also show that the flows always approach equilibrium in a very thin layers on the surface because the local velocity approaches zero. The density gradient near the surface create thin layers of vorticity on the surface which is important to boundary layer stability.

Hornung ${ }^{[29]}$ showed that the degree of nonequilibrium for hypersonic flow over a cylinder can be characteristized by a reaction rate parameter $\Omega$ defined by:

$$
\Omega=\left(\frac{d \alpha}{d t}\right) \frac{r}{U_{\infty}}
$$

where $d \alpha / d t$ is the dissociation rate of $N_{2}$ at the point immediate behind the shock, $U_{\infty}$ is free stream velocity. The parameter $\Omega$ represented the ratio of macroscopic time scale over dissociation time scale. Therefore, larger $\Omega$ represents more equilibrium flow. Figure 6 shows the calculated $\Omega$ distribution along the stagnation line for the steady solutions of the three test cases. 
As expected, Case $\mathrm{C}$ has much larger $\Omega$ value behind the shock than Case A and Case B do.

\section{Bow-Shock/Acoustic-Wave Interaction}

After the steady state solutions of the three cases have been obtained, we impose planer acoustic waves with a single wave number to the steady numerical solutions of the free steam, and advance the transient flow calculations in time. The planer incident acoustic wave of a single wave-length are:

$$
\Delta q=\delta q e^{i \bar{k}\left\{x-\left(u_{1}+c_{1}\right) t\right\}}
$$

where $q$ can be $u, v, p$, or $\rho$, and,

$$
\begin{array}{ll}
\delta u=\epsilon_{0} c_{1} & \delta v=0 \\
\delta p=\rho_{1} c_{1} \delta u & \delta \rho=\delta p / c_{1}{ }^{2}
\end{array}
$$

where $\epsilon_{0}=0.01$.

The wave fields of perfect-gas shock/disturbance interaction has been studied in [16]. In this paper, we investigate the real-gas effects on the interaction wave fields. In the simulations, for each of the three cases of steady flows, a number transient cases are computed with different free stream wave numbers, where the nondimensional wave number $k$ is normalized by the standoff distance as follows:

$$
k=\frac{\bar{k} d}{2 \pi}=\frac{d}{L}
$$

where $d$ is the standoff distance of the mean flow, $\bar{k}$ is the dimensional wave number, and $L$ is the wave length of the incident acoustic wave. For example, 23 transient test cases with freestream nondimensional wave numbers spanning the interval of $k=0.0452$ to $k=0.452$ are computed for Case C. The response of the wave fields to free stream wave length is studied in two aspects: the effects of nonequilibrium relaxation on the interaction wave fields and the possibility of perturbation resonant perturbed by incident acoustic waves. We mainly show the results of Case $\mathrm{A}$ and Case $\mathrm{C}$ due to length limitation of the paper.

\section{Space-Time Focusing of Entropy Waves}

It has been shown in [16] that due to the nonuniformity of the background mean flow fields, there is the space-time focusing of the entropy and vorticity waves on the body surface for wave fields behind the bow shock for perfect gas flow. Such space-time focusing is shown in Fig. 2, which is a schematic of acoustic and entropy wave patterns and trajectories along the stagnation line in the $x$ - $t$ plane. The bow shock/freestream wave interaction creates acoustic, entropy, and vorticity waves behind the shock. When the generated acoustic wave impinges on the wall, it generates a reflected acoustic wave which is propagated upstream. The entropy wave is propagated with a flow velocity, which decreases to zero at the wall. This nonuniform velocity field leads to curved entropy-wave lines, which do not reach the wall and are "packed" together near the wall. As a result, the local entropy wavelength decreases to zero as the entropy wave approaches the wall. This space-time focusing creates a singularity in the solutions of the Euler equations for the entropy and vorticity wave fields at the stagnation point. Consequently, there is a range of wavelengths for entropy and vorticity waves approaching the wall. These characteristics of the disturbance wave fields may play important roles in the receptivity process of hypersonic boundary layers.

In this paper, the real gas effects on the space-time focusing of the inviscid wave fields are investigated by studying perturbation contours in the $x$ - $t$ space along the stagnation line for the transient cases.

Figures 8 and 9 show the instantaneous pressure perturbation contours in the $x-t$ plane along the stagnation line for the same nondimensional wave number of $k=0.226$ with Case $\mathrm{A}$ and Case $\mathrm{C}$ as mean flows, where Case $\mathrm{A}$ is more nonequilibrium than Case C. Figure 8 shows that the pressure perturbations related to acoustic wave behind the shock are almost not affected by the nonequilibrium effects. The nonequilibrium real-gas effects on the entropy waves behind the shock are much stronger. The stronger nonequilibrium relaxation of Case $\mathrm{C}$ smears the entropy waves as shown in Fig. 9. For this case, the space-time focusing of the entropy wave is clearly seen in the results for Case C. The space-time focusing for the entropy wave become less severe for Case A due to nonequilibrium relaxation effects. This trend is more clear as the free stream wave number $k$ is even smaller.

Figures 10 and 11 show the instantaneous pressure perturbation contours in the $x$ - $t$ plane for a much smaller $k=0.0452$. With such a small wave number, the entropy wave fronts are much more smeared for Case $\mathrm{A}$ because the incident acoustic wave has smaller period in time so that the internal relaxation effects become more important. Because of the nonequilibrium effects, the entropy wave is dissipated near the wall. As a result, singular entropy wave solutions in the perfect 
flows and the small length scales due to the space-time focusing are smeared away by the relaxation. On the other hand, Case $\mathrm{C}$ still has much stronger space-time focusing effects at the stagnation point because the flow is near equilibrium for Case $\mathrm{C}$.

Therefore, nonequilibrium effects of the flow introduce dissipation to the flow fields and reduce the effects of the singularity of space-time focusing of the entropy waves near the wall.

\section{Vibrational Length Scales}

Figures 12 and 13 show instantaneous vibrational temperature perturbation contours in the $x$ - $t$ plane along the stagnation line for the case of $k=0.226$. The corresponding contours for the case of $k=0.0452$ are shown in Figs. 14 and 15. For Case A with high wave number of $k=0.226$, Fig. 12 shows that the interaction of the incident acoustic wave with the bow shock creates complex wave structure for vibrational temperature which is related to vibration relaxation of the flows. The figures show that new short length scales are introduced to the wave fields. One possible reason is that these new length scales are related to the vibrational relaxation for nonequilibrium flow behind the shock. On the other hand, there is not new length scale in the results for Case $\mathrm{C}$ in Figs. 12 to 13, because the flow is near equilibrium for Case $\mathrm{C}$, where the effects of the vibrational relaxation is not significant. The results of 14 and 15 have much longer wavelength than the vibration length scale, therefore the effects of vibrational relaxation is not visible in those figures.

\section{Effect of $k$ on Maximum Shock Oscillations}

A number of transient cases of different $k$ are computed to determine the values of $k$ such that the bow shock oscillation are maximum and minimum. Figures 22 and 23 show the RMS pressure perturbation contours of the test cases along the stagnation line for different value of freestream wave number $k$, for Case $\mathrm{C}$ and Case A respectively. The figures show that as the incident $k$ changes, there are values of $k$ corresponding to maximum and minimum shock oscillations. However, there is no resonance for disturbance wave fields between the shock and the body.

Figure 24 shows the RMS pressure perturbations at stagnation point as a function of $k$. Each point in the plot corresponds to the calculation of a transient test case with a given value of $k$. For Case A with nonequilibrium mean flow, the peak magnitude of the pressure perturbations are smaller than Case $\mathrm{C}$ due to internal relaxation, and the span of $k$ between two peaks increases for Case A.

Root-Mean-Square Values of the Perturbations

Figures 16 and 17 show the RMS contours of the entropy perturbations. Again, the figures show that for Case $A$, the nonequilibrium relaxation reduce the small wave lengths of entropy waves near the wall as compared to the more equilibrium Case C. For Case C, there is a reduction of local entropy wavelengths near the wall due to the space-time focusing of the entropy waves on the wall.

Figures 18 to 21 are the root-mean-square values of pressure and density perturbations along the stagnation line for $k=0.226$ and $k=0.0452$. Again, the results show that the pattern of pressure perturbations are not sensitive to nonequilibrium effects. The density length scales are smeared by the nonequilibrium effects in Case C.

\section{Approximate Linear Analysis for Wave Transfer Relations}

We apply the approach of McKenzie and Westphal ${ }^{[6]}$ to the interaction of a freestream planer disturbance wave with a local section of the curved bow shock for equilibrium reactive flow behind the bow shock. In [16], it was found such analysis is a good approximation for initial wave interaction before the reflected waves reach back to the bow shock.

The flow consists of real gas conditions both in front of and behind the shock. From the given free stream conditions, three separate flow characteristics were used to determine the mean flow properties behind the shock. These were equilibrium flow, chemically frozen flow, and chemically and vibrationally frozen flow. In the analysis, the equation of state is formulated as follows:

$$
P=(1+\alpha) \rho R T
$$

where $P$ is the pressure, $T$ is the temperature, $\rho$ is the density, $R$ is the gas constant of molecular nitrogen, and $\alpha$ is the mass fraction of atomic nitrogen. The internal energy and enthalpy are formulated by summing the contributions of the atomic nitrogen and molecular nitrogen and including the dissociation energy. For a diatomic species :

$$
\begin{aligned}
& e=\frac{3}{2} R T+e_{r}+e_{v} \\
& e_{r}=R T \\
& e_{v}=\frac{\theta_{v} R}{e^{\theta_{v} / T_{v}}-1}
\end{aligned}
$$


and for an atomic species :

$$
e=\frac{3}{2} R T
$$

This leads to total internal energy and enthalpy equations :

$$
\begin{aligned}
& e=3 R T \alpha+(1-\alpha)\left(\frac{5}{2} R T+\frac{\theta_{v} R}{e^{\theta_{v} / T_{v}}-1}\right)+\alpha R \theta_{d}(20) \\
& h=\left(\frac{7}{2}+\frac{3}{2} \alpha\right) R T+(1-\alpha)\left(\frac{\theta_{v} R}{e^{\theta_{v} / T_{v}}-1}\right)+\alpha R \theta_{d}(21)
\end{aligned}
$$

where $\theta_{v}$ is the characteristic temperature for vibration, and $\theta_{d}$ is the characteristic temperature for dissociation. Along with the temperature rise across the shock, there also is a dissociation of the diatomic nitrogen as well. The flow behind the shock must be solved numerically. For simplicity, the ideal dissociating gas model of Lighthill's ${ }^{[30]}$ is used in order to determine the nitrogen dissociation behind the shock. It is :

$$
\frac{\alpha^{2}}{1-\alpha}=\frac{\rho_{d}}{\rho} e^{-\theta_{d} / T}
$$

where $\rho_{d}$ is the characteristic density for dissociation. The remaining equations that are needed to calculate the mean flow properties come from the Rankine Hugoniot relations.

The calculation of the generated perturbation values is determined based on the method given by Mckenzie and Westphal ${ }^{[6]}$. By using real gas equations along with the Mckenzie/Westphal method, the perturbations can be determined.

Figure 28 shows the pressure perturbation across a normal shock for the three test cases with different cylinder radiuses. The numerical results are compared with linear analysis results for the frozen and equilibrium limits. The results show that the analysis is accurate using the current model.

The linear analysis is then used to studied the general trend of the wave transfer relations when free stream Mach number changes. As can be seen in Fig. 26, the increase in Mach number shows a corresponding increase in pressure perturbations when nondimensionalized with the free stream values. It can also be seen that the perturbation generated in equilibrium conditions is the largest. When non-dimensionalized with the local values, the pressure perturbation is shown to slightly decrease with increasing Mach number, again with the equilibrium condition producing the highest perturbation value. From Fig. 27, it is shown that the density perturbations will rise with increasing Mach number. This is regardless of whether it is non-dimensionalized with free stream or local values. There is a difference, however, in that the equilibrium condition produces a higher perturbation when nondimensionalized with the free stream values, whereas the frozen condition produces a higher perturbation value when non-dimensionalized with the local values.

\section{Approximate Analysis on Wall Effects}

Morkovin ${ }^{[31]}$ analyzed the effects of the body to the wave field along the stagnation line by assuming the mean flow variables between the shock wave and the body are uniform. The waves behind the shock are the combination of downstream propagating waves (acoustic and entropy waves) and upstream propagating acoustic wave reflected from the wall. The wave magnitudes and phase angle are solved using the linearized jump conditions at the shock and a velocity boundary condition at the wall. It was shown in [16] that such analysis is qualitatively correct for pressure fields only. In this paper, we extend the approach of Morkovin $^{[31]}$ to analyze the effects of the body to the pressure perturbation field along the stagnation line to equilibrium flows.

Figure 28 shows the maximum and minimum amplitudes of pressure perturbations along the stagnation line with the body effects for incident acoustic wave using Morkovin's analysis. The free stream conditions are the same as those used in the simulations in this paper. Since the pressure-perturbation amplitudes with the body effects are functions of $x$ along the stagnation line, only their maximum and minimum values are plotted in the figure. The figure shows that amplitudes of the pressure perturbations with the presence of the body are about two times as large as the amplitude without body effects as shown in Fig. 26. As the Mach number increases, the perturbations induced by the incident acoustic wave increases for the equilibrium flow case while the corresponding values for frozen flows decreases.

\section{Conclusions}

The real gas effects on the interaction of freestream disturbance waves with a bow shock in hypersonic flow past a cylinder have been studied in this paper. 
The freestream disturbances are fixed-frequency planar acoustic waves. The nonequilibrium real-gas effects have been investigated by the numerical simulations of two-dimensional Euler equations and by two approximate linear analyses. The results show :

1. Unlike ideal gas flow where there is a space-time focusing of the entropy and vorticity waves on the wall, the nonequilibrium real gas effects dissipate away small length-scale entropy and vorticity waves near the body. Consequently, the spacetime focusing of the entropy waves is less severe for nonequilibrium flows at the stagnation point.

2. The nonequilibrium vibration relaxation introduces new short length scales to the perturbation wave fields when the mean flows are nonequilibrium and when the incident wavelengths are at the same order as the vibrational length scales.

3. There is no resonance in shock oscillations induced by freestream waves, but there are incident wave numbers corresponding to maximum shock oscillations. The real gas effects reduce to peak values of such maximum oscillations.

4. The approximate linear analysis for reactive flows compares well with numerical simulations in the limited comparisons made so far.

\section{Acknowledgements}

This research was supported by the Air Force Office of Scientific Research under grant numbers F4962094-1-0019 and F49620-95-1-0405 monitored by Dr. Len Sakell. More information on this work and related ongoing research can be found on the World Wide Web at http://cfdlab5.seas.ucla.edu.

\section{References}

[1] M. V. Morkovin. Critical evaluation of transition from laminar to turbulent shear layer with emphasis on hypersonically traveling bodies. Air Force Flight Dyn. Lab. Rep., AFFDL-TR-68-149, 1969.

[2] L. S. G. Kovasznay. Turbulence in supersonic flow. Journal of the Aeronautical Sciences, 20(10):657682, October 1953.

[3] H. S. Ribner. Convection of a pattern of vorticity through a shock wave. NACA TR 1164, 1954.
[4] C. T. Chang. On the Interaction of Weak Disturbances and a Plane Shock of Arbitrary Strength in a Perfect Gas. PhD thesis, Johns Hopkins University, 1955.

[5] C. T. Chang. Interaction of a plane shock and oblique plane disturbances with special reference to entropy waves. Journal of Aeronautical Sciences, 24:675-682, September 1957.

[6] J. F. Mckenzie and K. O. Westphal. Interaction of linear waves with oblique shock waves. The Physics of Fluids, 11(11):2350-2362, November 1968.

[7] H. S. Ribner. Shock-turbulence interaction and the generation of noise. NACA TR 1233, 1954.

[8] J. C. Anyiwo and D. M. Bushnell. Turbulence amplification in shock-wave boundary-layer interaction. AIAA Journal, 20(7):893-899, July 1982.

[9] S. K. Lele. Shock jump conditions in a turbulence flow. Physics of Fluids: A, 4:2900-2905, 1994.

[10] T. A. Zang, M. Y. Hussaini, and D. M. Bushnell. Numerical computations of turbulence amplification in shock-wave interactions. AIAA Journal, 22(1):13-21, January 1984.

[11] M. Y. Hussaini, F. Collier, and D. M. Bushnell. Turbulence alteration due to shock motion. Turbulent Shear Layer/Shock Wave Interactions, IUTAM Symp., Ed. J. Delery, Springer, pages 371$381,1986$.

[12] D. Rotman. Shock wave effects on a turbulent flow. The Physis of Fluids A, 3(7):1792-1806, July 1991.

[13] S. Lee, S. K. Lele, and P. Moin. Interaction of isotropic turbulence with a strong shock wave. AIAA Paper 94-0311, 1994.

[14] S. K. Lele. Compressibility effects on turbulence. Annual Review of Fluid Mechanics, 26:211-254, 1994.

[15] C.-H. Chiu and X. Zhong. Numerical Simulation of Transient Hypersonic Flow Using the Essentialy Nonoscillatory Schemes. AIAA Journal, Vol. 34, No. 4, pp. 655-661, 1995. (Also AIAA paper 95$0469,1995)$.

[16] X. Zhong, X. Joubert, and T. K. Lee. The interaction of freestream disturbances and the bow shock in hypersonic flow past a cylinder. 1995. Presented in 20th International Symp. on Shock Waves, July 23-28, 1995, Pasadena, California, Also submitted to Journal of Fluid Machanics. 
[17] M. T. Landahl. Wave mechanics of breakdown. Journal of Fluid Mechanics, Vol. 56, No. 4, pp. 775-802, 1972 .

[18] H. Hornung. Experimental simulation of highenthalpy real-gas effects. in Hypersonic Flows for Reentry Problems, Eds. J.-A. Desideri, $R$. Glowinski, and J. Periaux, Springer-Verlag, 1:187-208, 1991.

[19] M. R. Malik. Stability theory for chemically reacting flows. In D. Arnal and R. Michel, editors, Laminar-Turbulent Transition, IUTAM Symp., Toulouse, Springer-Verlag, 1990.

[20] G. K. Stuckert and H. L. Reed. Stability of hypersonic, chemically reacting viscous flows. AIAA Paper 90-1529, 1990.

[21] M. L. Hudson, N. Chokani, and G. V. Candler. Linear stability of hypersonic flow in thermochemical nonequilibrium. AIAA Paper 96-0671, 1996.

[22] X. Zhong. New High-Order Semi-implicit RungeKutta Schemes for Computing Transient Nonequilibrium Hypersonic Flow. AIAA Paper 95-2007, 1995.

[23] C. Park. On convergence of chemically reacting flows. AIAA Paper 85-0247, 1985.

[24] G. Candler. On the computation of shock shapes in nonequilibrium hypersonic flows. AIAA Paper 89-0312, 1989

[25] R. C. Millikan and D. R. White. Systematics of vibrational relaxation. J. of Chemical Physics, 39(12):3209-3213, 1963.

[26] C. Shu and S. Osher. Efficient implementation of essentially non-oscillatory schemes II. Contractor Report 181656 ICASE Report No. 88-24, NASA, April 1988.

[27] A. Harten, B. Engquist, S. Osher, and S. Chakravarthy. Uniformly High Order Accurate Essentially Non-oscillatory Schemes, III. Journal of Computational Physics, Vol. 71, No. 2, pp. 231-303, 1987.

[28] J. Casper. Essentially Non-Oscillatory Shock Capturing Schemes to Multi-Dimensional Systems of Conservation Laws. PhD thesis, Old Dominion University, December 1990.

[29] H. G. Hornung. Non-equilibrium dissociating nitrogen flow over spheres and circular cylinders. Journal of Fluid Mechanics, 53:149-176, 1972.

[30] M. J. Lighthill. Dynamics of a dissociating gas. part i. equilibrium flow. Journal of Fluid Mechanics, 2(1):1, 1957.
[31] M. V. Morkovin. Note on the assessment of flow disturbances at a blunt body traveling at supersonic speeds owing to flow disturbance in free stream. J. Applied Mech., pages 223-229, 61960. 


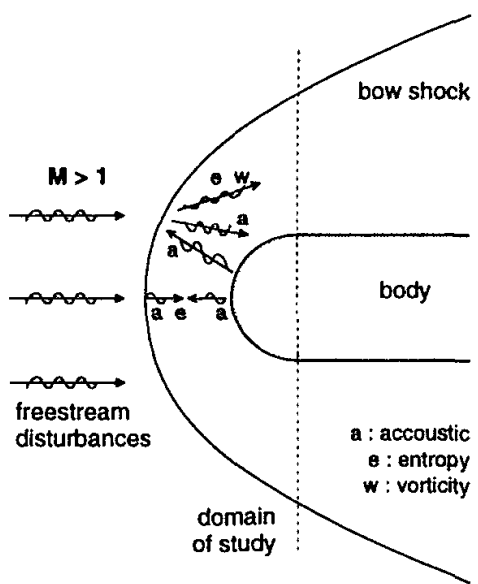

Figure 1: A schematic of the wave field of the interaction between the bow shock and free-stream disturbances.

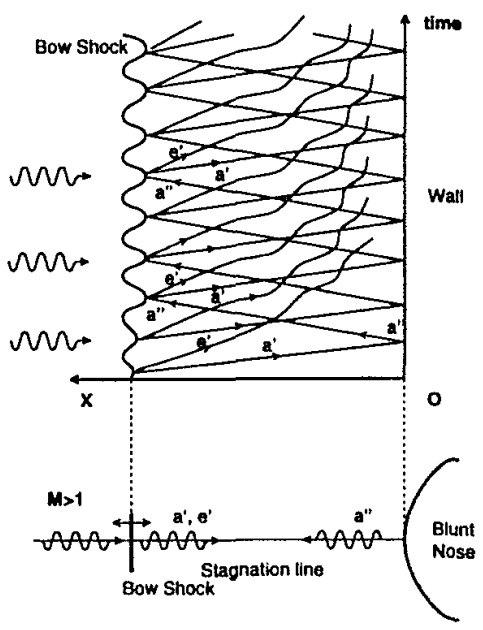

Figure 2: A schematic of acoustic and entropy wave patterns and trajectories along the stagnation line in the $x$-t plane (a: acoustic wave, e: entropy wave).

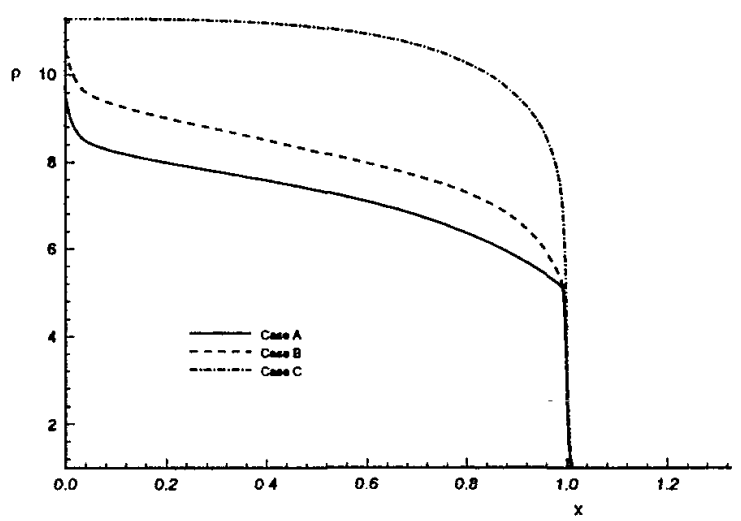

Figure 3: Steady density $\left(\mathrm{kg} / \mathrm{m}^{3}\right)$ distributions along the stagnation line for the three test cases.

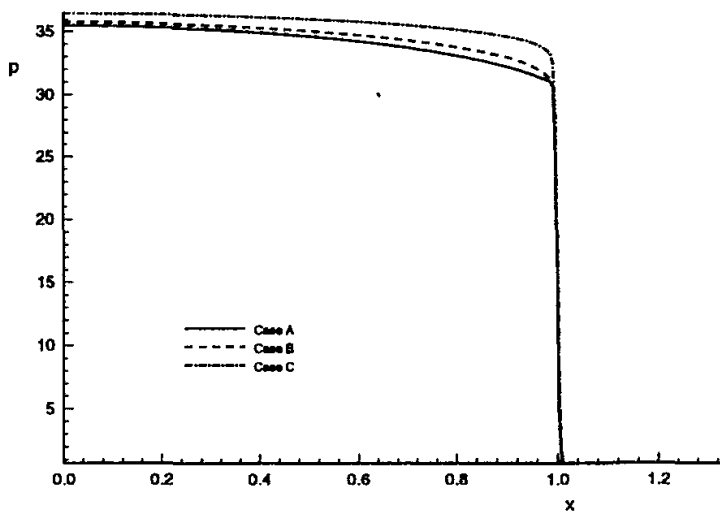

Figure 4: Steady pressure $(\mathrm{Pa})$ distributions along the stagnation line for the three test cases.

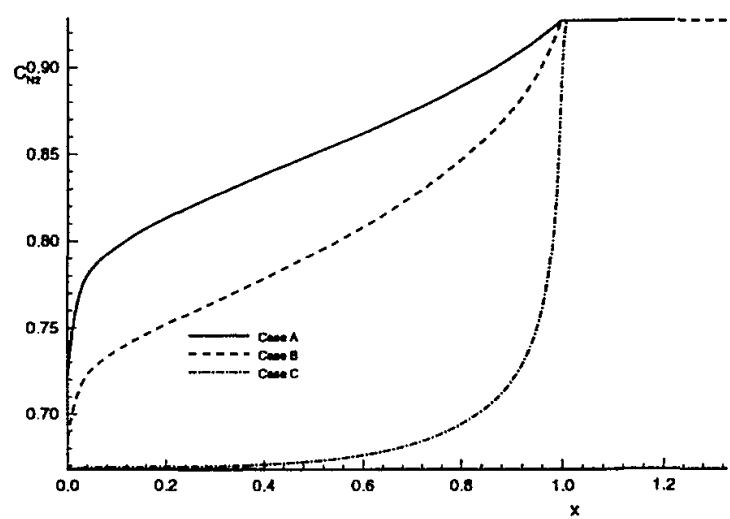

Figure 5: Steady mass fraction of $N_{2}$ distributions along the stagnation line for the three test cases.

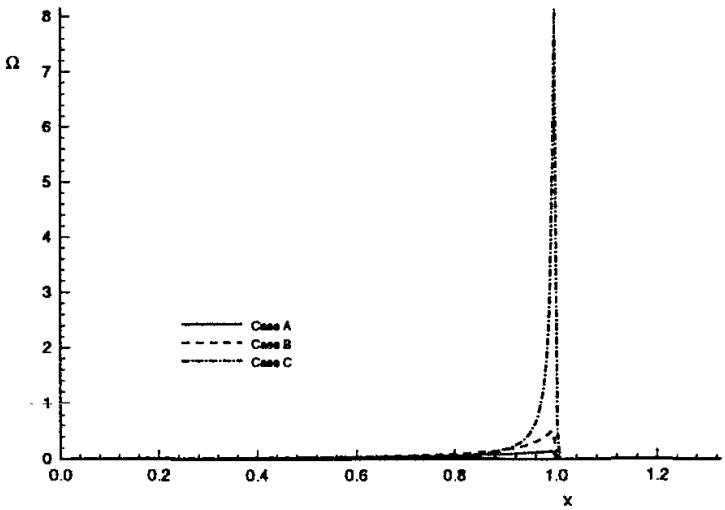

Figure 6: Steady nonequilibrium factor along the stagnation line for three test cases. 
Copyright (C)1996, American Institute of Aeronautics and Astronautics, Inc.

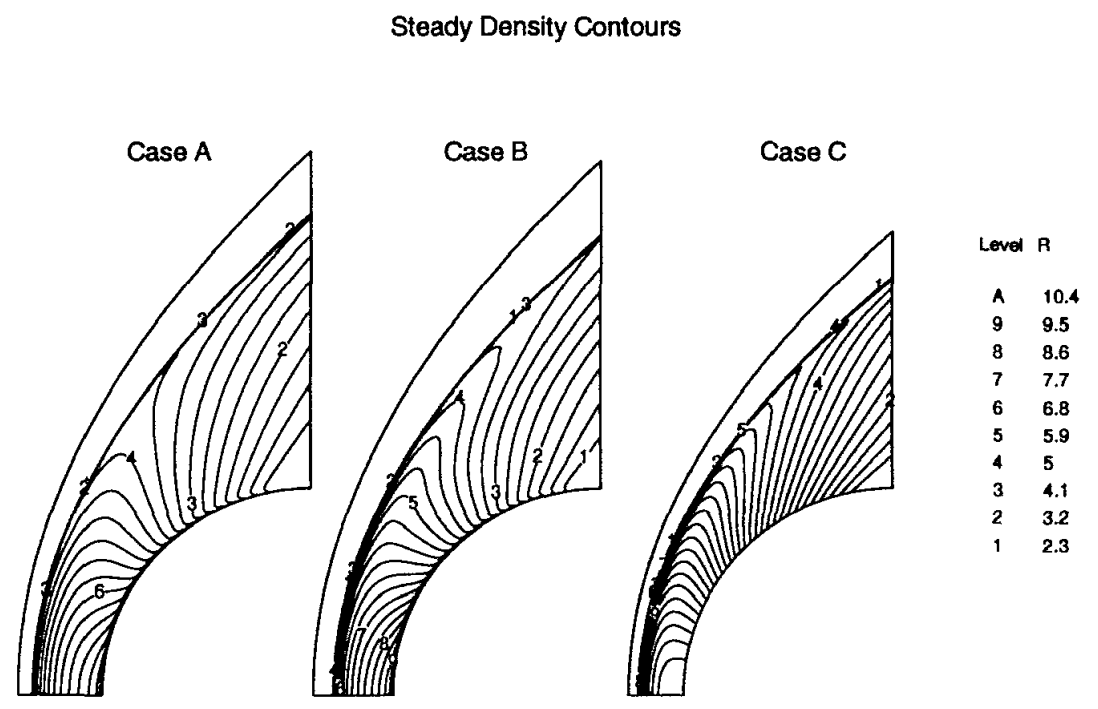

Figure 7: Steady density contours of the mean-flow solutions for three test cases with different levels of nonequilibrium. 


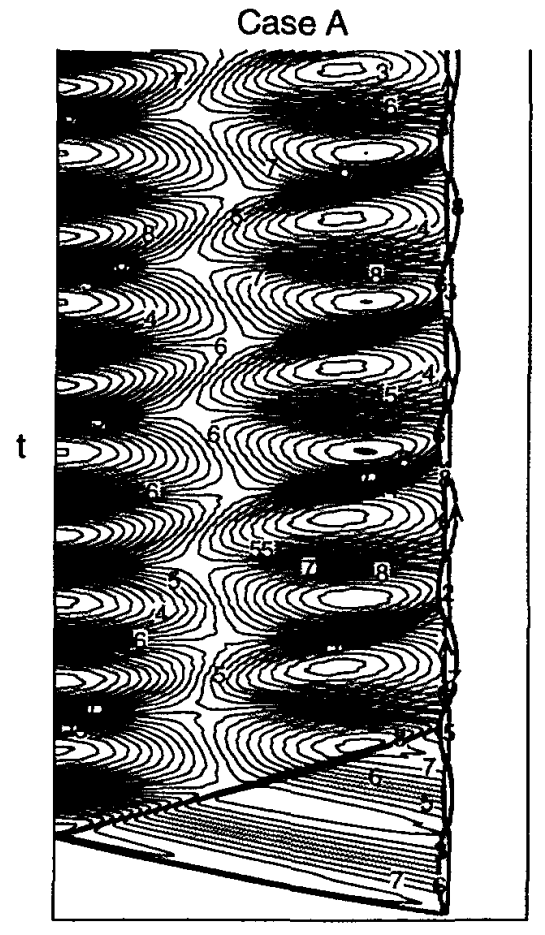

$\mathbf{X}$

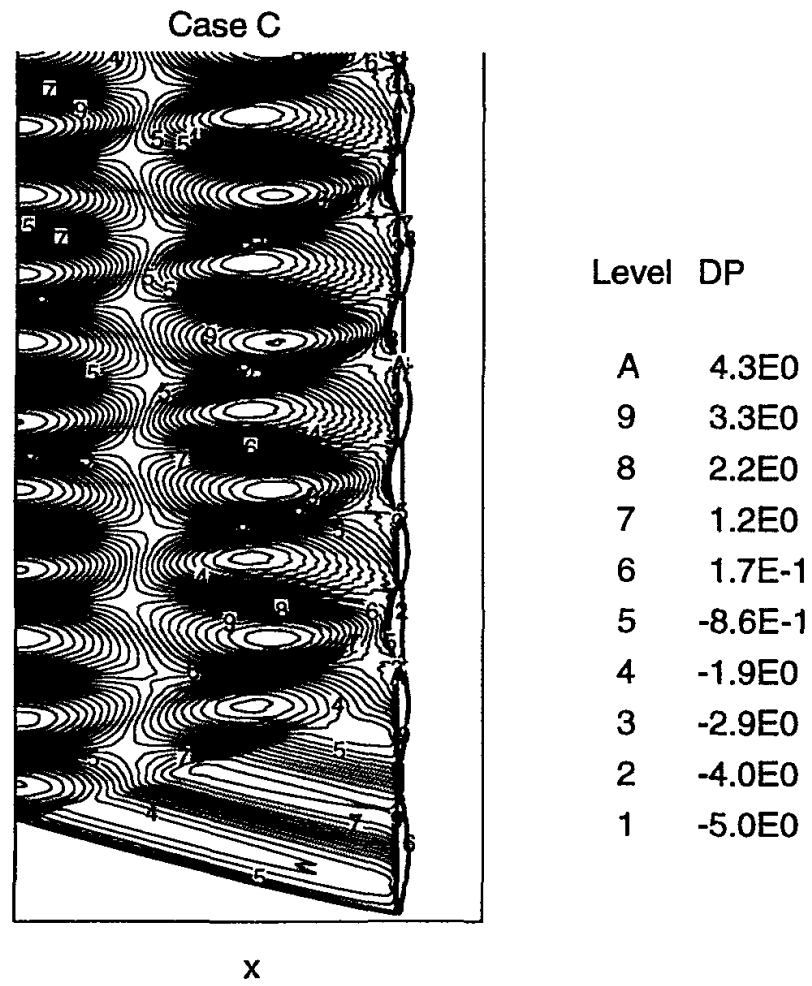

Figure 8: Instantaneous pressure perturbation contours in the $x-t$ plane along the stagnation line $(k=0.226)$.

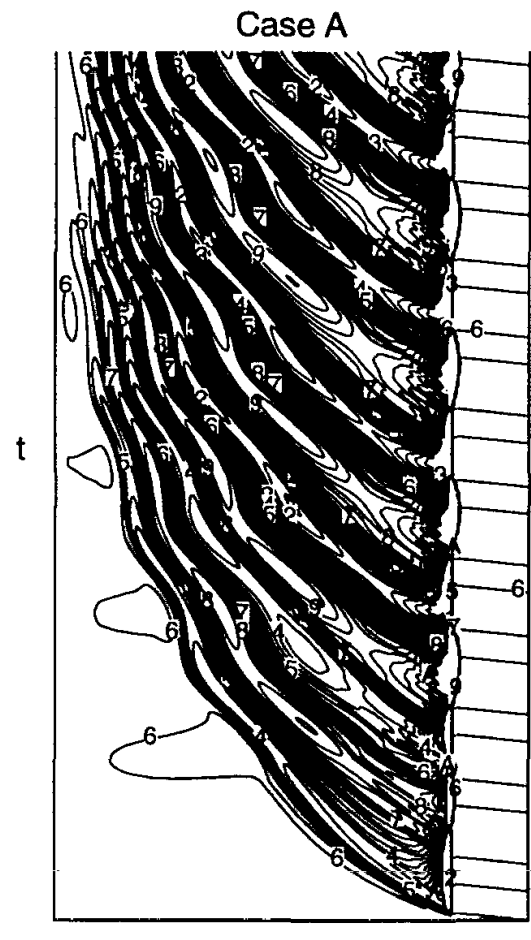

$\mathbf{X}$

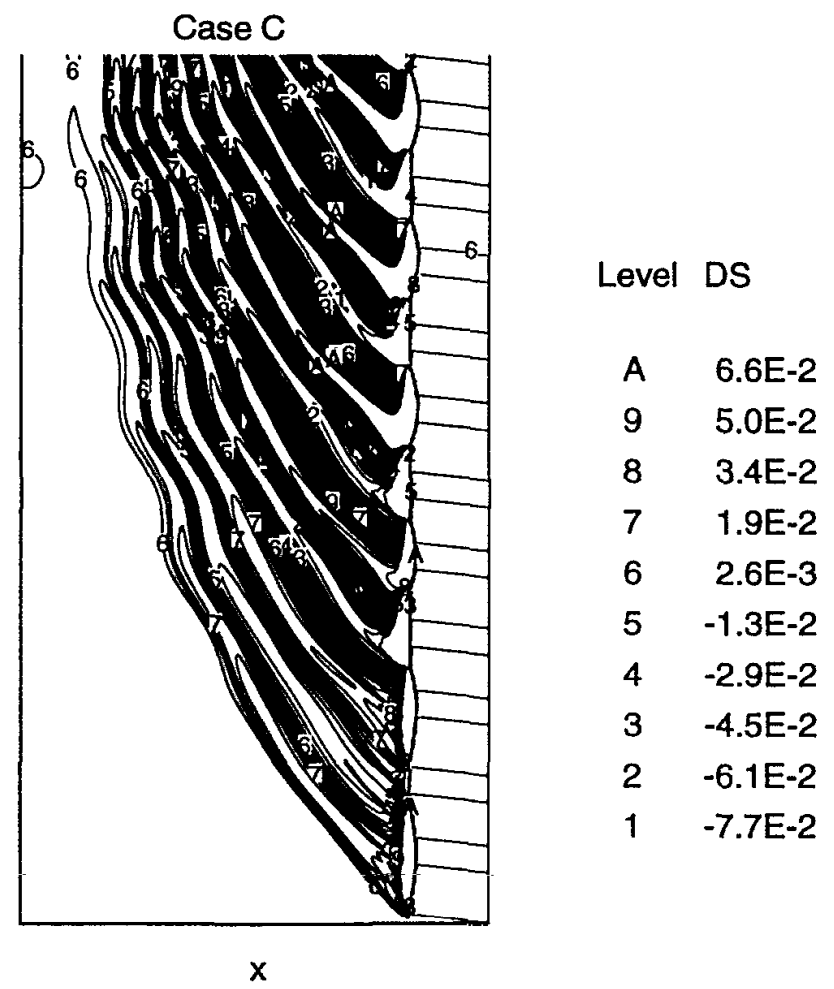

Figure 9: Instantaneous entropy perturbation contours in the $x$ - $t$ plane along the stagnation line $(k=0.226)$. 
Case A

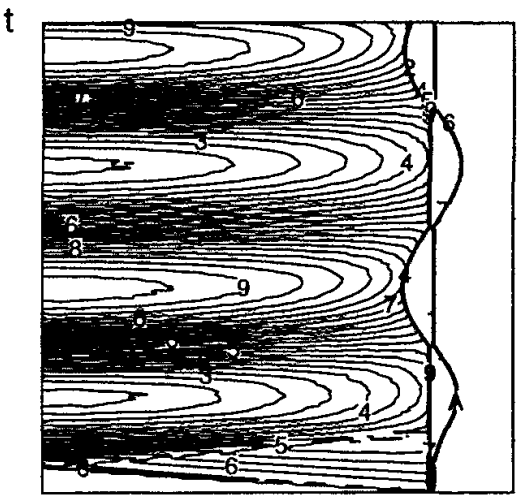

$\mathbf{X}$
Case C

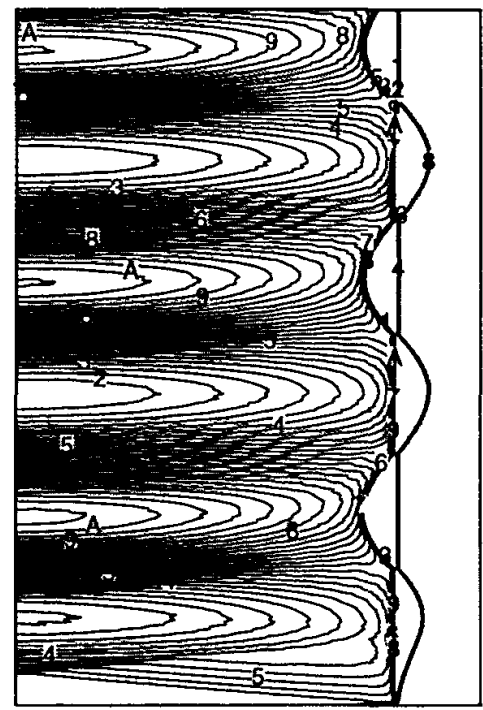

$x$
Level DP

A 4.3E0

9 3.3E0

8 2.2E0

7 1.2E0

6 1.7E-1

5 -8.6E-1

$4-1.9 E 0$

$3-2.9 E 0$

2 -4.0EO

1 -5.0EO

Figure 10: Instantaneous pressure perturbation contours in the $x-t$ plane along the stagnation line $(k=0.0452)$.

Case A

\section{$t$}

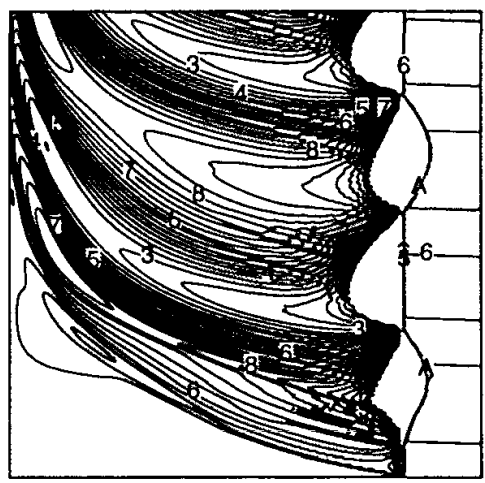

$\mathrm{x}$
Case C

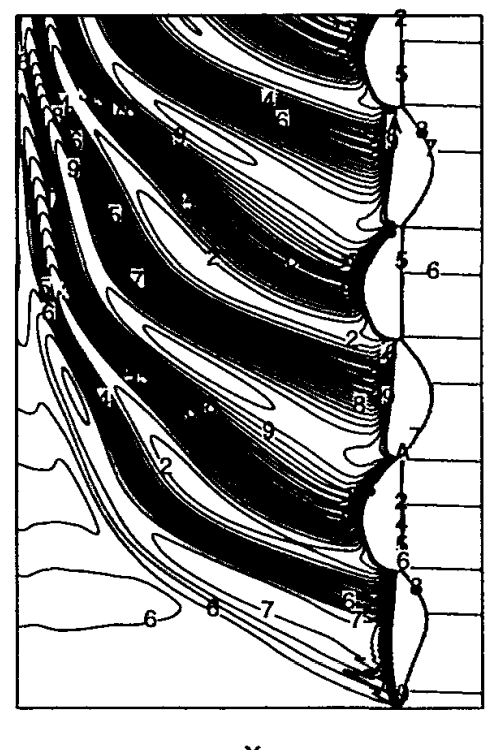

Level DS

A $7.1 \mathrm{E}-2$

9 5.4E-2

8 3.7E-2

$7 \quad 2.0 \mathrm{E}-2$

$6 \quad 2.8 \mathrm{E}-3$

$5 \quad-1.4 E-2$

$4-3.1 E-2$

$3-4.8 \mathrm{E}-2$

$2-6.5 \mathrm{E}-2$

$1-8.2 E-2$

Figure 11: Instantaneous entropy perturbation contours in the $x-t$ plane along the stagnation line $(k=0.0452)$. 


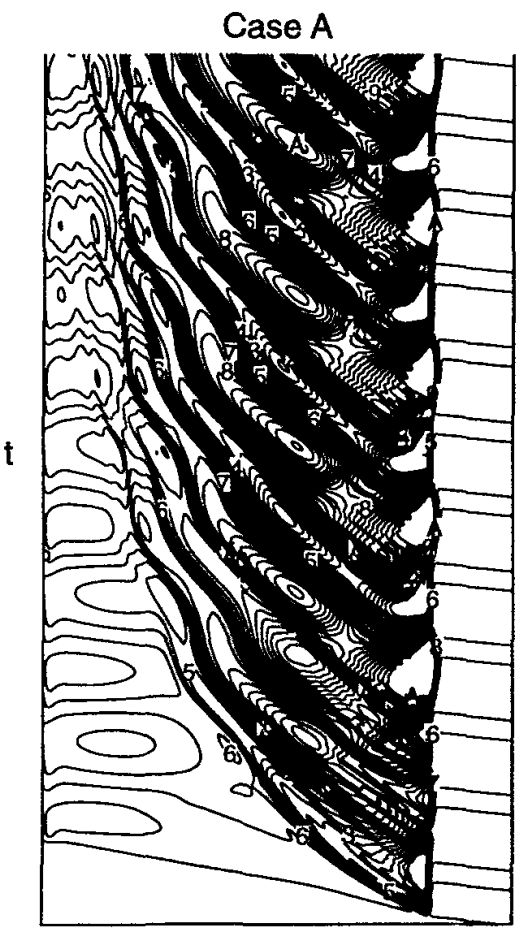

$x$

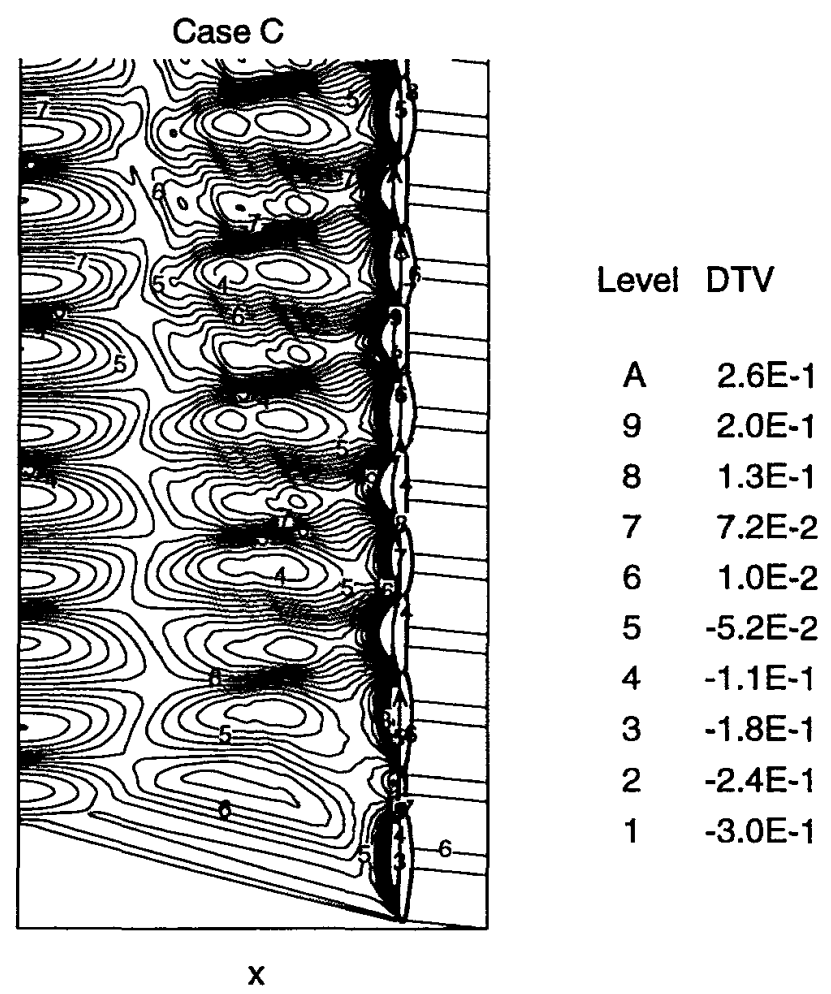

Figure 12: Instantaneous $T_{v}$ perturbation contours in the $x$-t plane along the stagnation line $(k=0.226)$.
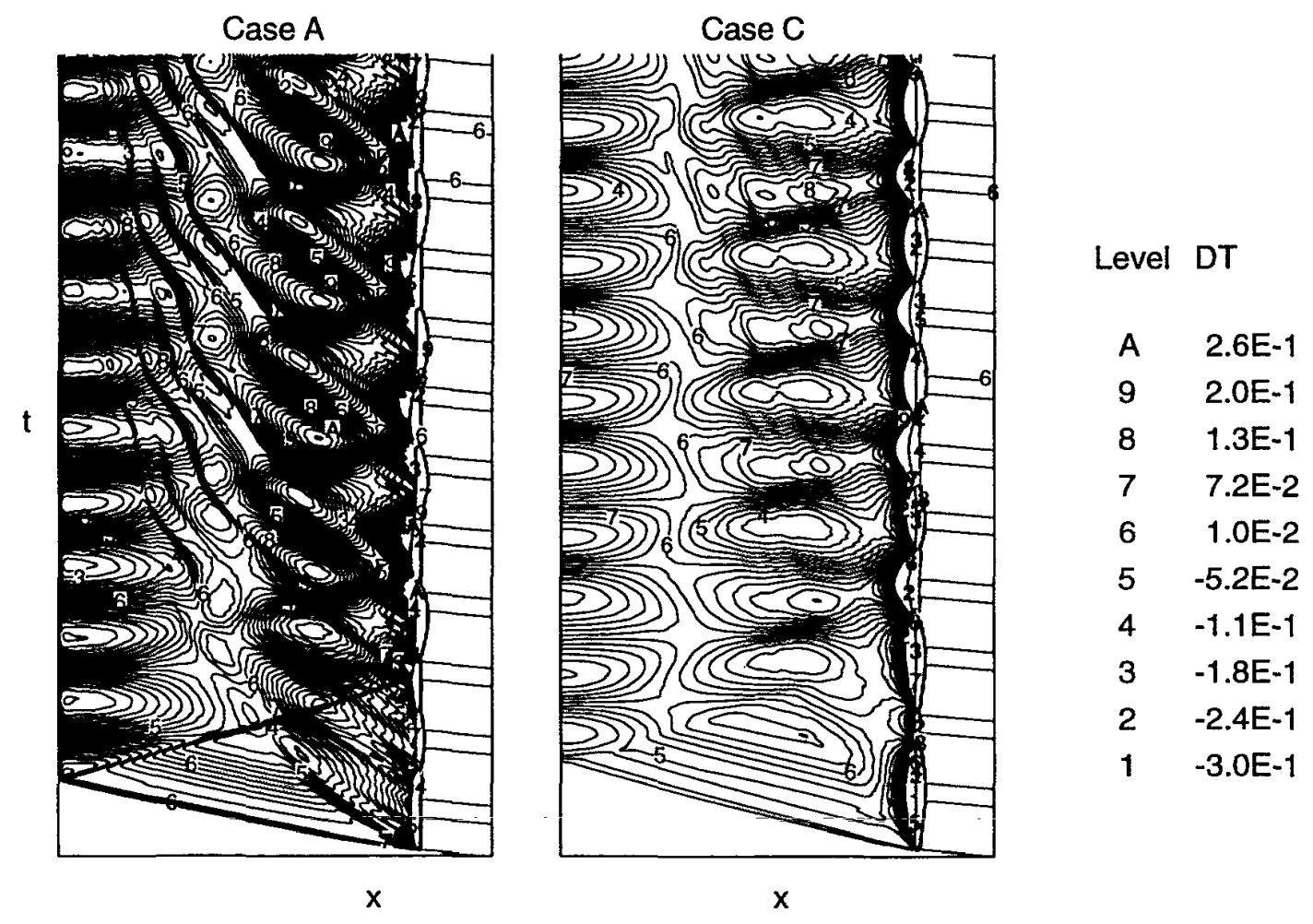

Figure 13: Instantaneous $T$ perturbation contours in the $x-t$ plane along the stagnation line $(k=0.226)$. 
Case A

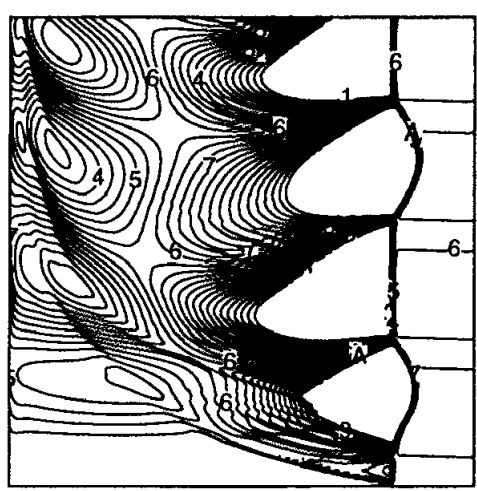

$\mathrm{x}$
Case C

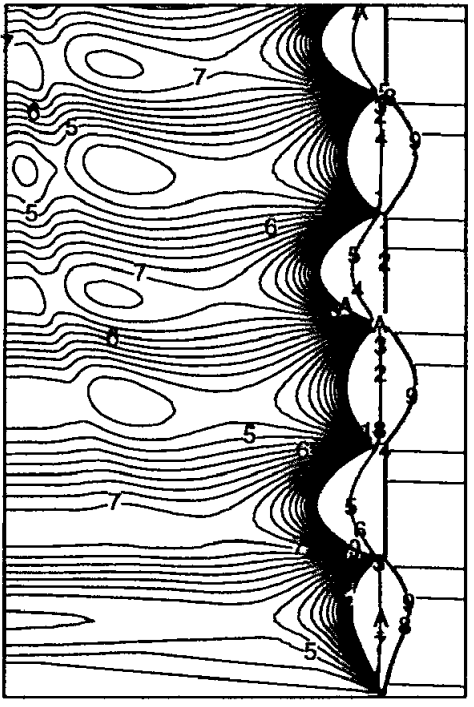

X
Level DTV

A $2.6 \mathrm{E}-1$

9 2.0E-1

8 1.3E-1

7 7.2E-2

6 1.0E-2

5 -5.2E-2

4 -1.1E-1

3 -1.8E-1

2 -2.4E-1

$1-3.0 \mathrm{E}-1$

Figure 14: Instantaneous $T_{v}$ perturbation contours in the $x$ - $t$ plane along the stagnation line $(k=0.0452)$.

Case A

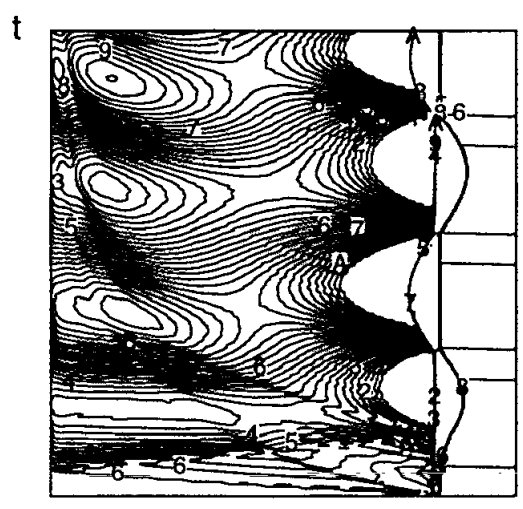

$x$
Case C

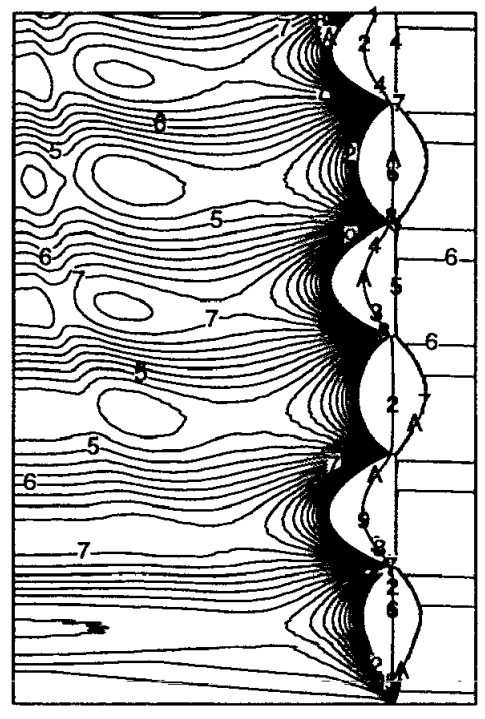

$x$
Level DT

$\begin{array}{ll}\text { A } & 2.6 \mathrm{E}-1 \\ 9 & 2.0 \mathrm{E}-1 \\ 8 & 1.3 \mathrm{E}-1 \\ 7 & 7.2 \mathrm{E}-2 \\ 6 & 1.0 \mathrm{E}-2 \\ 5 & -5.2 \mathrm{E}-2 \\ 4 & -1.1 \mathrm{E}-1 \\ 3 & -1.8 \mathrm{E}-1 \\ 2 & -2.4 \mathrm{E}-1 \\ 1 & -3.0 \mathrm{E}-1\end{array}$

Figure 15: Instantaneous $T$ perturbation contours in the $x-t$ plane along the stagnation line $(k=0.0452)$. 


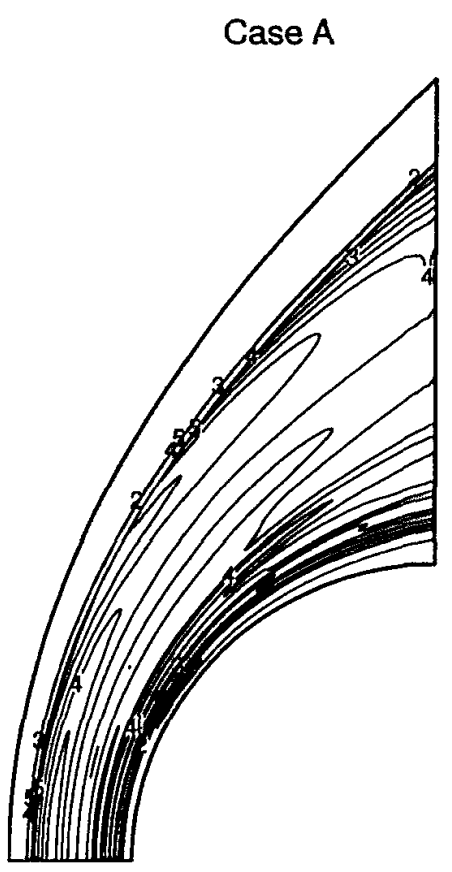

Case C

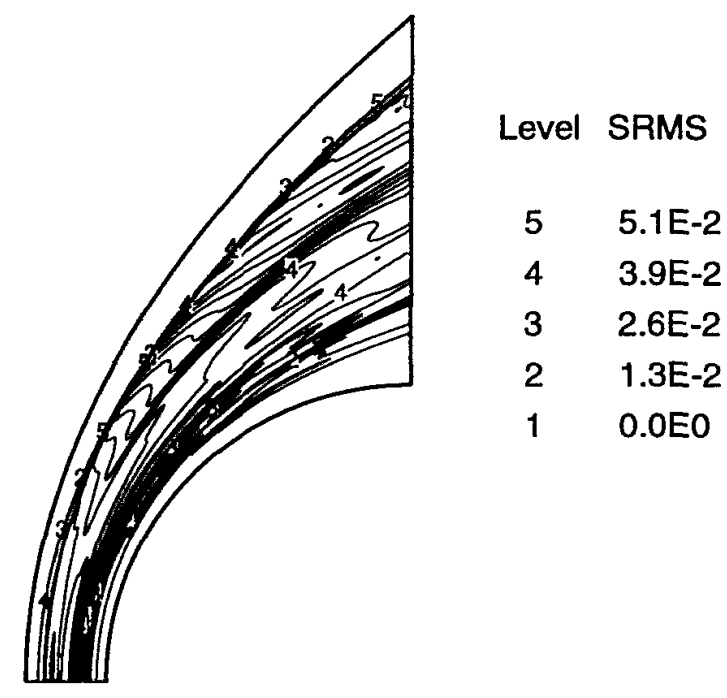

Figure 16: Root-mean-square pressure perturbation contours for the case of $k=0.226$.
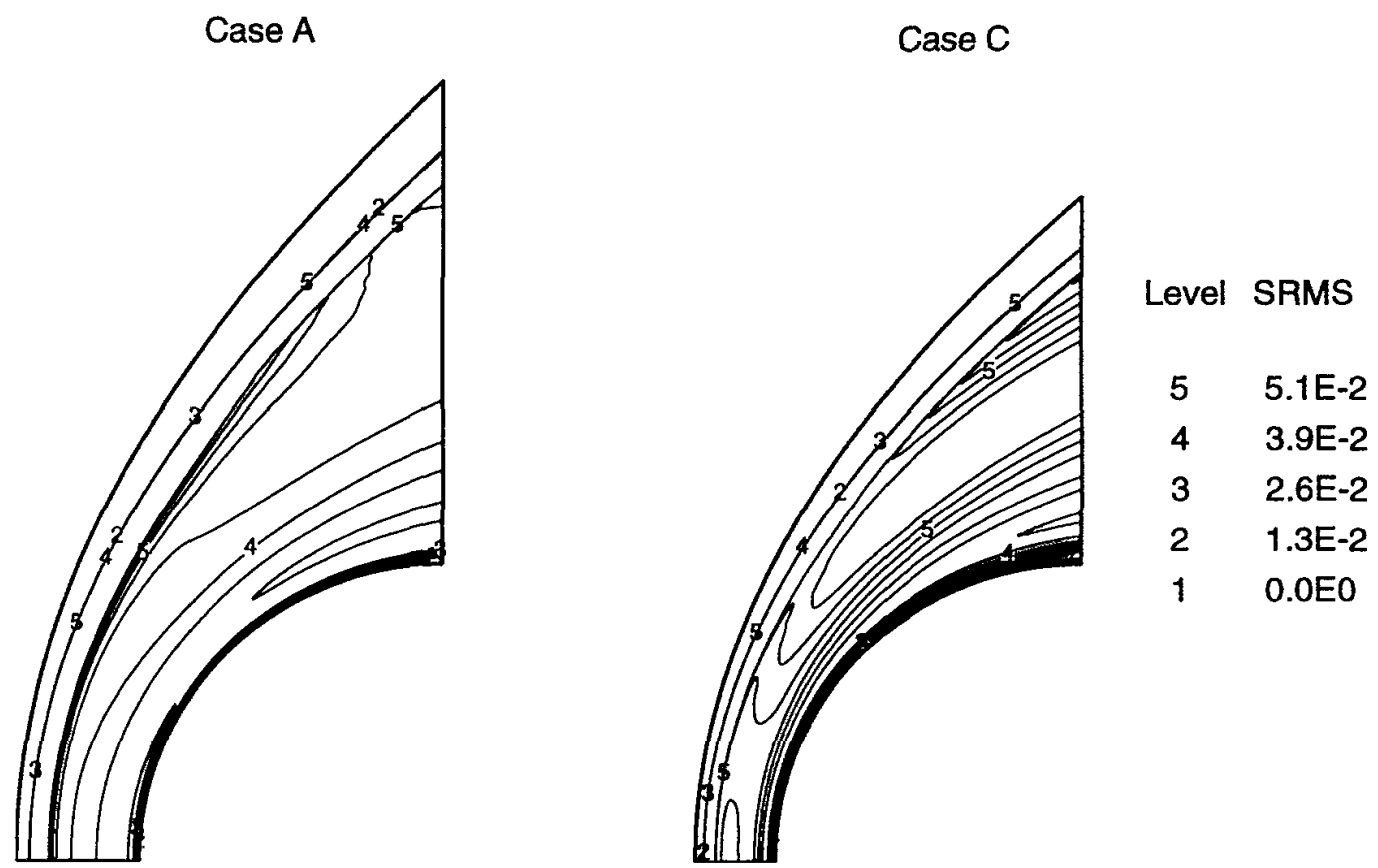

Figure 17: Root-mean-square pressure perturbation contours for the case of $k=0.0452$. 


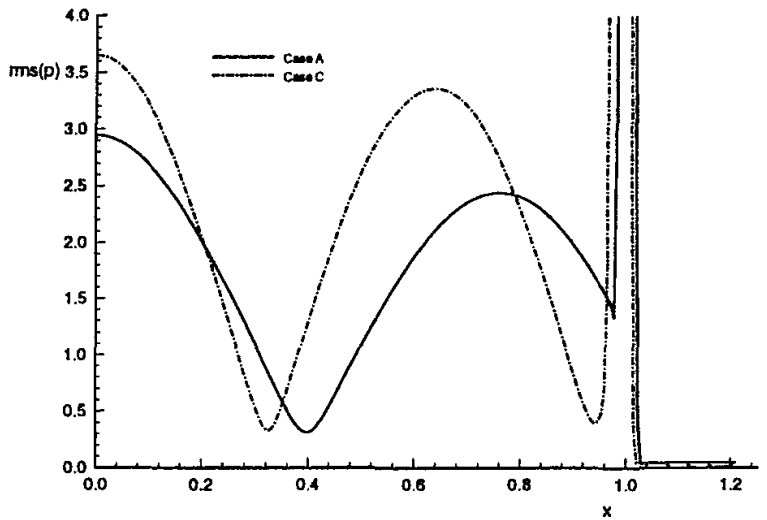

Figure 18: Root-mean-square values of pressure perturbations along the stagnation line for the case of $k=0.226$.

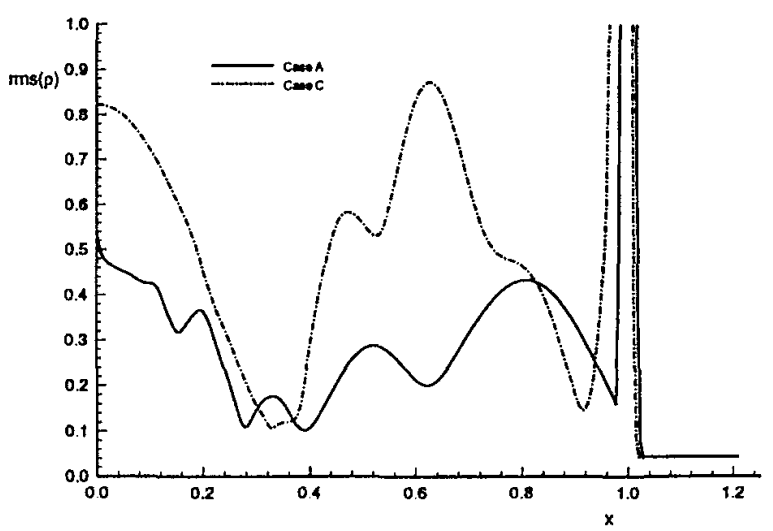

Figure 19: Root-mean-square values of density perturbations along the stagnation line for the case of $k=0.226$.

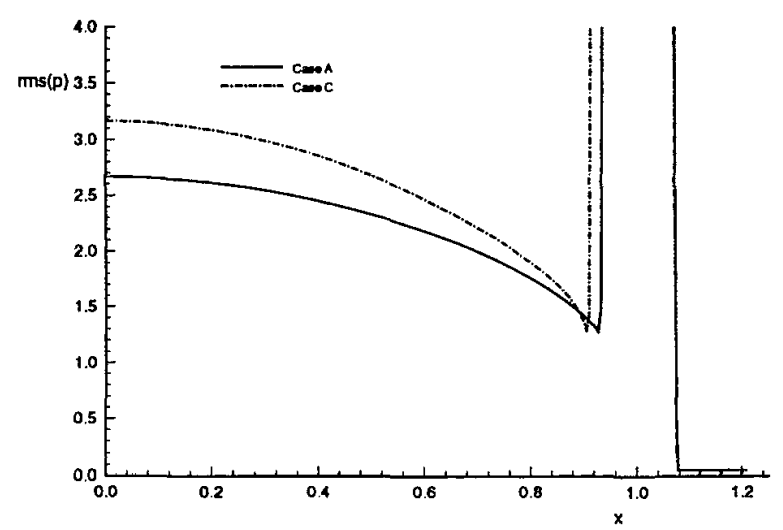

Figure 20: Root-mean-square values of pressure perturbations along the stagnation line for the case of $k=0.0452$.

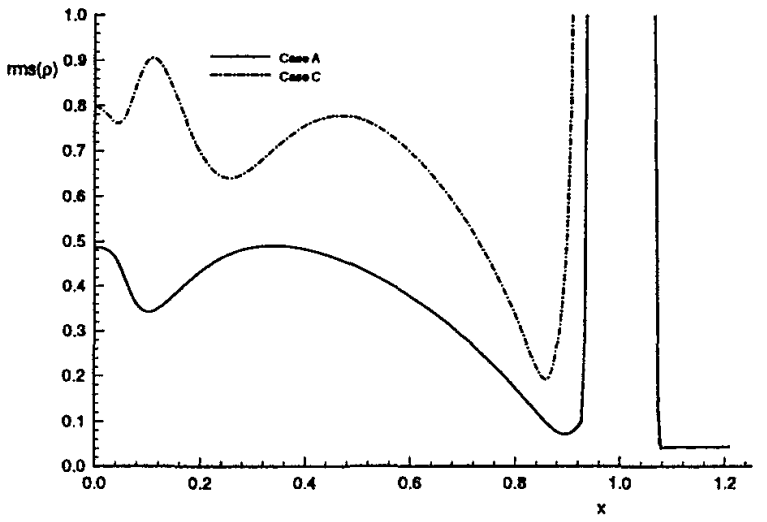

Figure 21: Root-mean-square values of density perturbations along the stagnation line for the case of $k=0.0452$.

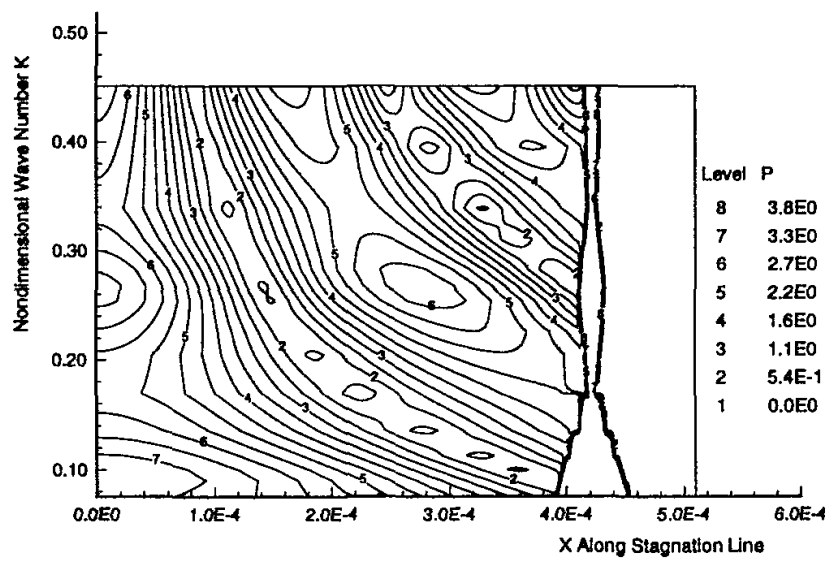

Figure 22: RMS pressure perturbation contours along the stagnation line for different value of freestream wave number $k$ for Case $A$.

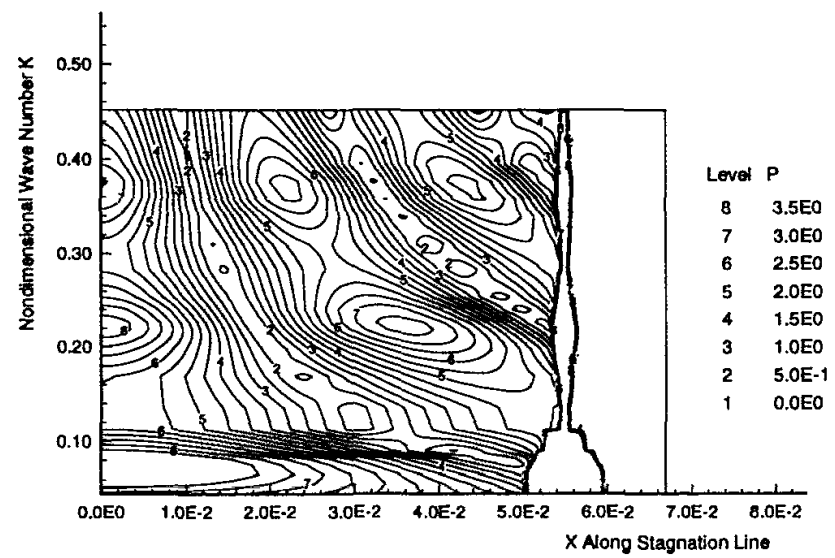

Figure 23: RMS pressure perturbation contours along the stagnation line for different value of freestream wave number $k$ for Case C. 


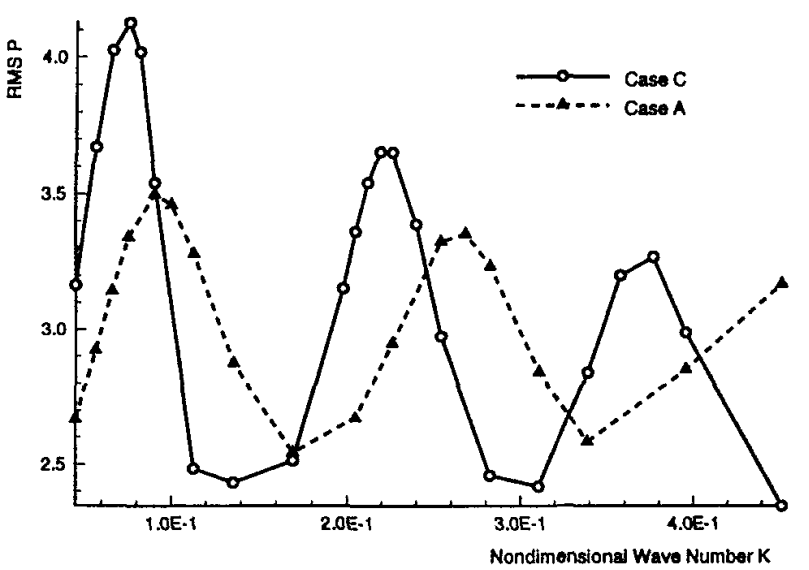

Figure 24: RMS pressure perturbations at stagnation point at test cases of different $k$.

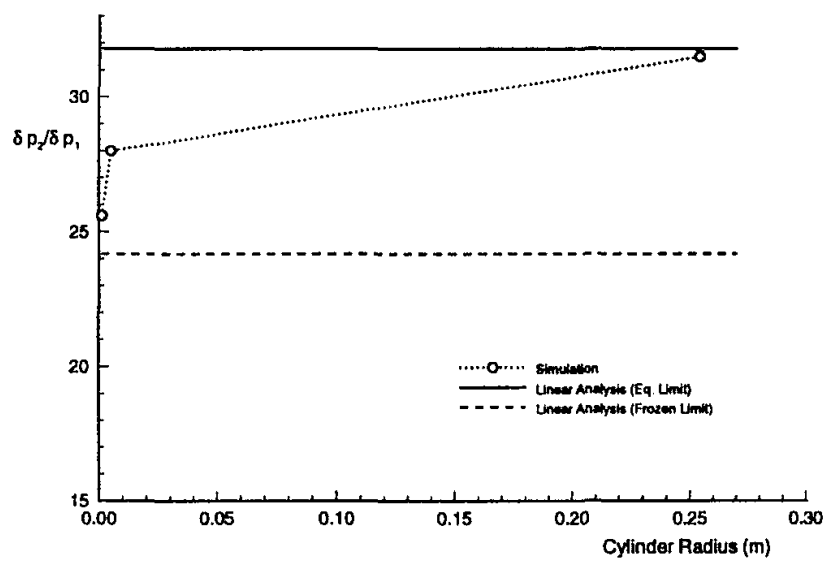

Figure 25: Pressure perturbation across a normal shock for the three test cases with different cylinder radiuses.

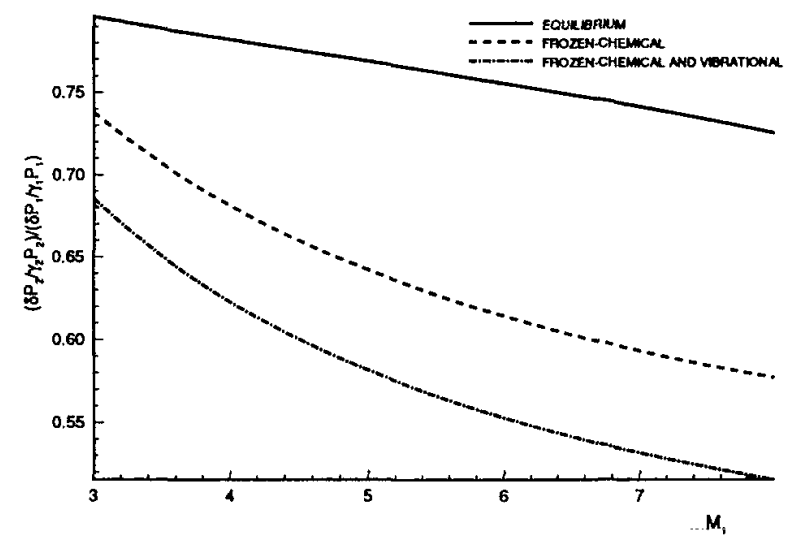

Figure 26: Pressure amplitude transfer coefficients for the pressure perturbations along the bow shock as a function of freestream Mach number with incident acoustic wave.

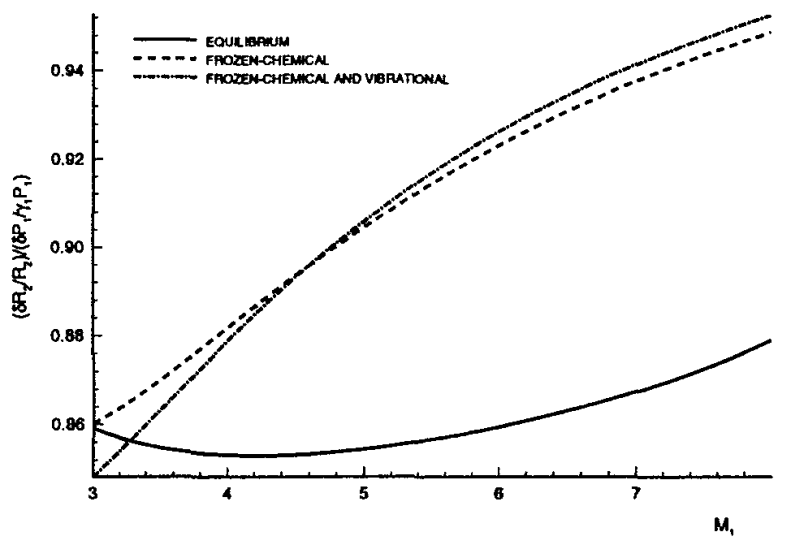

Figure 27: Density amplitude transfer coefficients for the pressure perturbations along the bow shock as a function of freestream Mach number with incident acoustic wave.

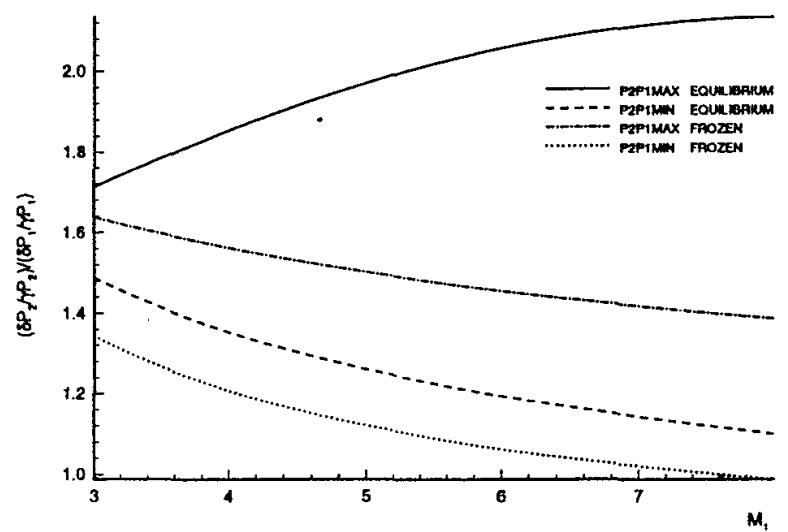

Figure 28: The maximum and minimum amplitudes of pressure perturbations along the stagnation line with the body effects for incident acoustic wave using Morkovin's analysis. 